\title{
Reversibility of MRI Features of Pseudotumor Cerebri Syndrome
}

\author{
J.A. Fraser, A.E. Leung
}

Can J Neurol Sci. 2014; 41: 530-532

An 18-year-old woman started oral minocycline 100mg daily for acne. Two months later, she presented to the emergency room with increasing headaches, worse with Valsalva maneuver and bending forward, and intermittent horizontal binocular diplopia when lying flat. She had no blurry vision, transient visual obscurations, or pulsatile tinnitus. Although overweight for several years, she had not had any recent weight gain.

Her past medical history was notable only for acne and stable asthma. Medications included minocycline, an oral contraceptive agent (ethinyl estradiol/levonorgestrel), vitamin $\mathrm{B}_{12}$, and montelukast sodium. Apart from her new use of minocycline, her medications had remained unchanged for four years. She did not use tobacco or alcohol. Family history was notable only for cluster headaches in her father.
She had a body mass index of $31.8 \mathrm{~kg} / \mathrm{m}^{2}$ (obese). Visual acuity was 20/20 in each eye. She saw 10/10 Ishihara colour plates with each eye. Pupillary examination was normal, and there was no relative afferent pupillary defect. No binocular misalignment was detected with alternate cover testing. Funduscopy revealed moderate optic disc edema bilaterally (Figure 1A). Neurological examination was normal. Humphrey visual field perimetry showed enlarged physiological blind spots (Figure 2A).

Cerebral magnetic resonance imaging / venography (MRI/ MRV) showed a partially empty sella turcica (Figure 3A), flattening of the posterior globes and intraocular protrusion of the optic nerve heads (Figure 3B), vertical tortuosity of the retrobulbar optic nerve and its sheath (Figure 3C), and non-

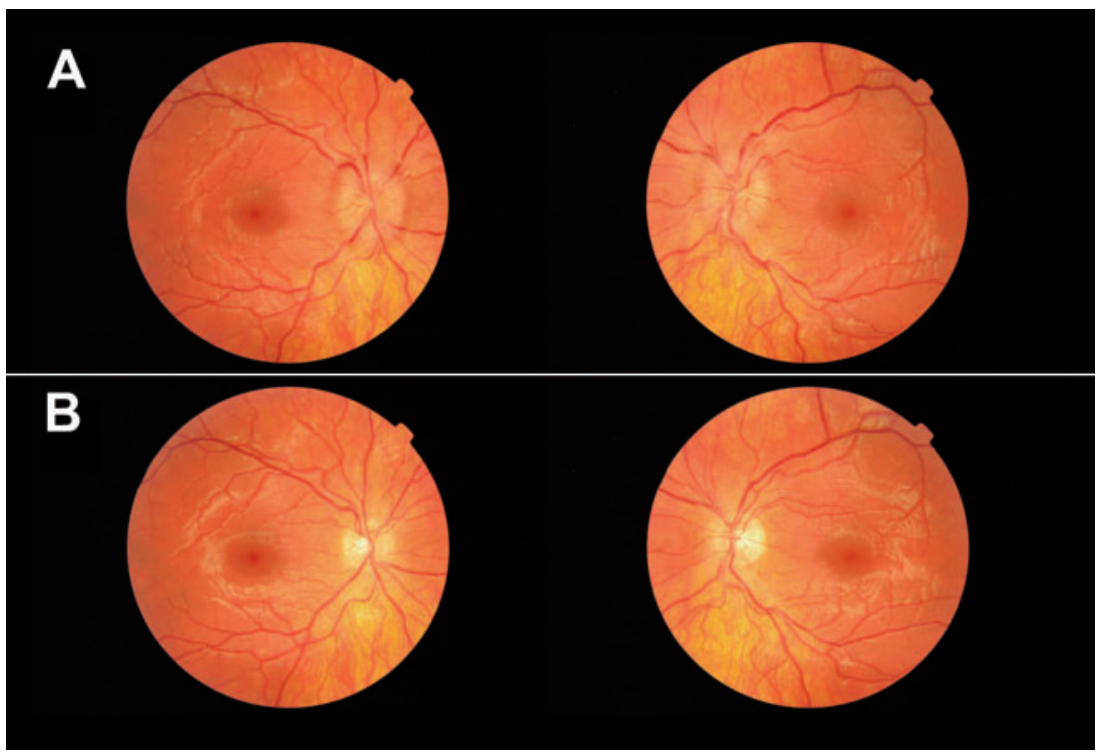

Figure 1: Ocular fundus appearance. A) Bilateral papilledema on initial presentation. B) Resolution of papilledema at 3-month follow-up visit.

\footnotetext{
From the Departments of Clinical Neurological Sciences (JAF), Ophthalmology (JAF), Medical Imaging (AEL), Western University, London, Ontario, Canada. Received November 19, 2013. Final Revisions Submitted January 13, 2014.

Correspondence to: J. Alexander Fraser, Western University, London Health Sciences Centre, University Hospital, Rm. B7-104, 339 Windermere Rd., London, Ontario N6A 5A5, Canada. Email: alex.fraser@lhsc.on.ca.
} 


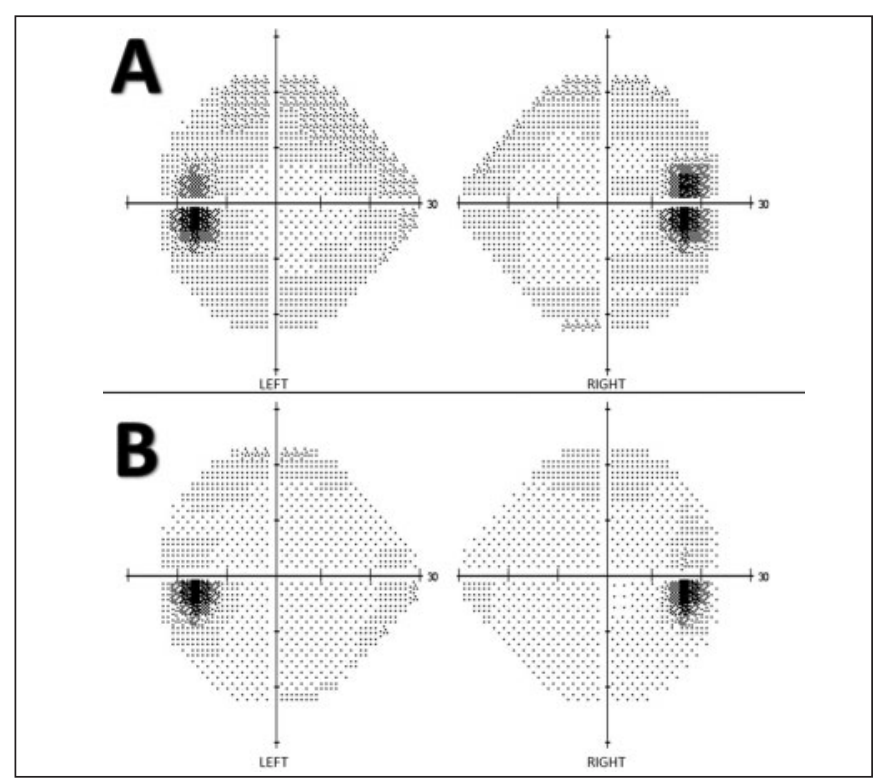

Figure 2: Humphrey visual field (grey-scale plot only). A) Enlarged blind spots on initial presentation. B) Resolution of enlarged blind spots at 3-month follow-up visit.

thrombotic distal transverse sinus stenoses (Figure 3D), consistent with pseudotumor cerebri syndrome (PTCS).

Lumbar puncture revealed a markedly elevated opening cerebrospinal fluid (CSF) pressure $\left(>55 \mathrm{~cm} \mathrm{H}_{2} \mathrm{O}\right)$ with normal CSF composition. She was diagnosed with PTCS secondary to minocycline use.

The patient's symptoms resolved immediately postprocedure, and the minocycline was discontinued. She was started on oral acetazolamide 500mg three times daily to avert a rebound increase in intracranial pressure (ICP) while the minocycline washed out of her system. Three months later, the patient remained asymptomatic, her papilledema and enlarged blind spots had resolved (Figures 1B, 2B), and her MRI/MRV showed complete normalization of all findings (Figure 3E-H).

\section{Discussion}

Minocycline is a lipophilic semisynthetic tetracycline derivative which can cause a syndrome of raised intracranial pressure (ICP) indistinguishable from the syndrome of idiopathic intracranial hypertension (IIH) ${ }^{1}$. Because minocycline-associated intracranial hypertension meets all of the 2002 diagnostic criteria for IIH, but because "idiopathic intracranial hypertension secondary to minocycline" is an oxymoron, updated 2013 diagnostic criteria include both minocycline-associated intracranial hypertension and IIH as variants of the newlyrevived entity of "pseudotumor cerebri syndrome" (PTCS)2.

These new criteria more explicitly recognize that patients with apparent IIH may actually have a medication-induced syndrome. When PTCS occurs in obese young women who start minocycline, such as our patient, one possibility is that the drug exacerbates an underlying tendency toward PTCS in patients who are already at risk for the IIH variant of PTCS, and tips them into a clinical syndrome. Such a syndrome would still be termed "minocycline-associated PTCS" according to the new 2013 diagnostic criteria, as the term "IIH" is now strictly reserved for primary, or idiopathic, PTCS ${ }^{2}$. (Although our patient was also using oral contraception, epidemiological studies have not shown a causal relationship between oral contraceptives and PTCS.)

The mechanism by which minocycline raises ICP is not known, but one theory holds that minocycline causes decreased CSF absorption and increased CSF outflow resistance at the level of the arachnoid villi, possibly due to its interference with cyclic AMP pathways ${ }^{1}$.

Minocycline-associated PTCS typically occurs within two months of starting (or increasing) the drug ${ }^{1}$, as it did with our patient, although delays as long as one year have been reported ${ }^{1}$. Minocycline-associated PTCS can be severe or even fulminant ${ }^{3}$. The associated papilledema and visual field defects range from

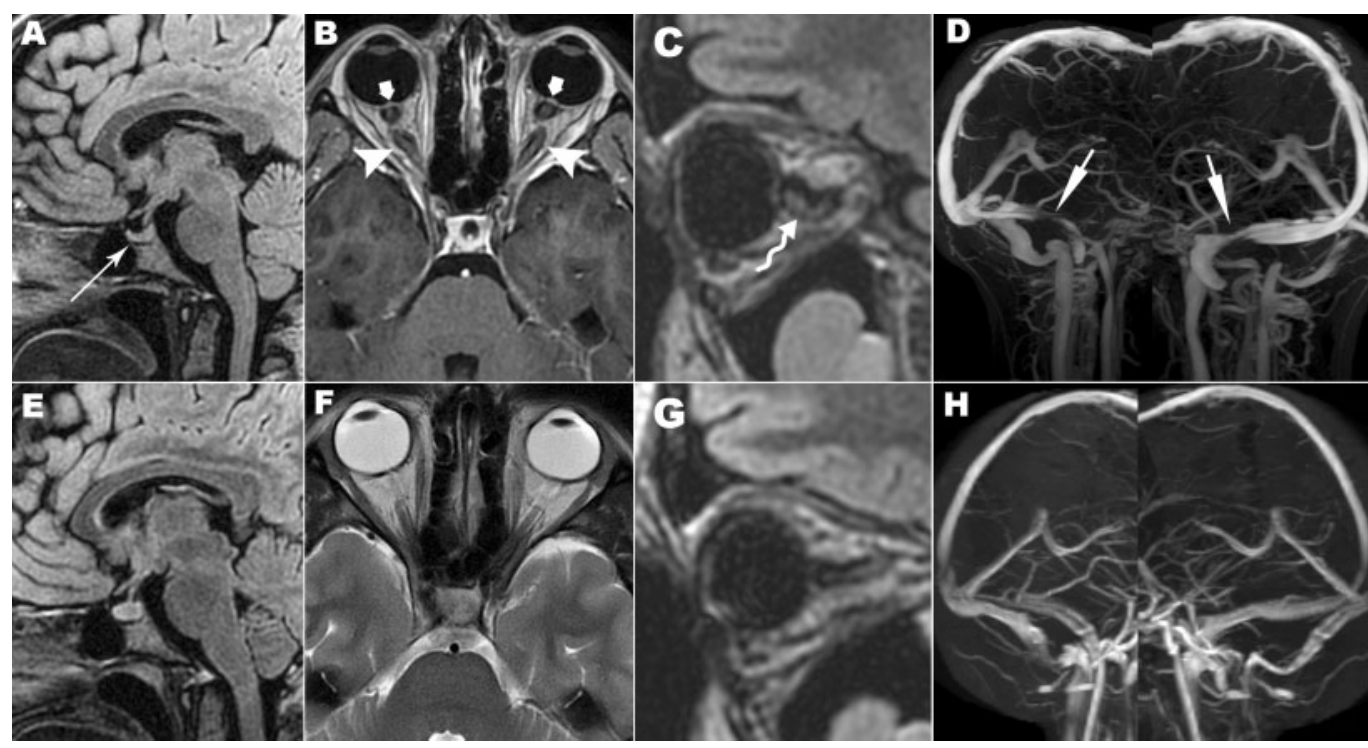

Figure 3: MRI/MRV findings of pseudotumor cerebri syndrome: A) Partially empty sella turcica (long arrow). B) Flattening of the posterior globes and intraocular protrusion of the optic nerve heads (short arrows); dilation of the optic nerve sheaths (arrowheads). C) Vertical tortuosity and kinking of the retrobulbar optic nerve and its sheath (twisty arrow). D) Distal transverse sinus stenoses (arrows). E, F,G,H) Complete normalization of all MRI/MRV findings three months later, after the lowering of intracranial pressure. 
Table: Main radiological features of pseudotumor cerebri syndrome

\begin{tabular}{c|cl}
\hline Imaging Modality & \multicolumn{2}{l}{ Finding } \\
\cline { 2 - 3 } & 1. & Partially empty sella \\
\hline MRI & 2. & Flattening of posterior globe \\
\hline & 3. & Intraocular protrusion of optic nerve head \\
\hline & 4. & Dilation of optic nerve sheath \\
\hline & 5. & Tortuosity of retrobulbar optic nerve and sheath \\
\hline MRV & 6. & Distal transverse sinus stenosis \\
\hline CT & 7. & Widened foramen ovale \\
\hline
\end{tabular}

mild to severe, and opening CSF pressures on lumbar puncture can be extremely high. Cessation of minocycline typically results in rapid resolution of the syndrome of raised ICP and no recurrence of the disease; however, when the optic nerves have been permanently injured from acute papilledema, visual field defects may persist ${ }^{1}$. In severe or fulminant cases, optic atrophy and severe permanent vision loss may occur ${ }^{3}$.

Pseudotumor cerebri, which can be asymptomatic on presentation in $25 \%$ of cases $^{4}$, has been associated with a number of statistically significant MRI findings ${ }^{5,6}$ (summarized in the Table), all of which our patient had. These include: 1) partially empty sella turcica, from increased and pulsatile intracranial pressure causing an arachnocele to herniate through the diaphragma sella and compress the pituitary gland; 2) flattening of the posterior globes, from increased CSF pressure behind the lamina cribrosa deforming the posterior sclera; 3) intraocular protrusion of the optic nerve head, from axoplasmic stasis and engorgement at the level of the optic disc related to compression of optic nerve axons from raised CSF pressure - the radiological manifestation of papilledema; 4) dilation of the optic nerve sheath, from increased CSF pressure "inflating" and stretching the sheath outwards; and 5) tortuosity or "kinking" of the retrobulbar optic nerve, from expansion of the optic nerve sheath against the fixed globe. One finding on MR venography, 6) distal transverse sinus stenosis, is more often seen in PTCS patients than in control subjects, but whether this finding is the effect of intracranial hypertension (causing focal collapse of the venous sinuses in areas of increased mural compliance) or the cause of intracranial hypertension (by impeding cerebral venous outflow and therefore decreasing CSF absorption) is currently the subject of intense study. Evidence so far suggests most patients have a combination of these two phenomena, with individual patients having different proportions of "cause" and "effect", depending on anatomic variability ${ }^{7}$. An additional finding in chronic PTCS, evident only on computed tomogram (CT) of the skull base, is that of: 7) a widened foramen ovale, from osseous erosion and remodeling in the setting of longstanding elevated CSF pressure $^{8}$.
Contrary to popular belief, small or "slit-like" lateral ventricles are not a distinctive radiological finding in PTCS, despite early CT and skull X-ray studies reporting such an association. Careful subsequent studies have refuted this association, showing no significant difference in ventricle size in PTCS patients compared to controls 9

The significant MRI/MRV findings described above all arise from the deforming effect of increased CSF pressure on adjacent intracranial structures. Several of these radiological findings have been individually reported to reverse following treatment of raised intracranial pressure; however, to our knowledge, our case is the first to exhibit the presence of, and subsequent complete reversal of, all recognized MRI/MRV findings of PTCS in a single patient. Our case proves that, at least in some patients, the characteristic MRI/MRV findings are due to elevated intracranial pressure itself and normalize when the ICP does. The reversal of the MRV findings in our patient may serve as a reminder to those who advocate indiscriminate stenting of venous sinus stenoses that such stenoses are sometimes the effect of, rather than the cause of, raised ICP.

Our patient had only been on minocycline for two months before presentation, and the short duration of her disease may have allowed the deformed CSF-adjacent structures to retain some elasticity and spring back to their original shapes after intracranial pressure was lowered. Not all patients have such a dramatic radiological response to correction of their intracranial hypertension, and patients with longer durations of raised ICP may develop more permanent distortions of tissues and less reversibility of their MRI/MRV findings. Although our patient had an excellent clinical course, visual outcomes in general do not correlate well with radiological findings. For this reason, we recommend that follow-up of patients with PTCS include neuroimaging as just one possible component of a broader approach which must also include clinical assessment, ophthalmoscopic examination, and automated visual field perimetry.

\section{REFERENCES}

1. Chiu AM, Cheunkongkaew WL, Cornblath WT, et al. Minocycline treatment and pseudotumor cerebri syndrome. Am J Ophthalmol. 1998;126:116-21.

2. Friedman DI, Liu GT, Digre KB. Revised diagnostic criteria for the pseudotumor cerebri syndrome in adults and children. Neurology. 2013;81:1159-65.

3. Fraser CL, Biousse V, Newman NJ. Minocycline-induced fulminant intracranial hypertension. Arch Neurol. 2012;69:1067-70.

4. Galvin JA, Van Stavern GP. Clinical characteristics of idiopathic intracranial hypertension at the Detroit Medical Center. J Neurol Sci. 2004;223:157-60.

5. Degnan AJ, Levy LM. Pseudotumor cerebri: brief review of clinical syndrome and imaging findings. Am J Neuroradiol. 2011;32: 1986-93.

6. Brodsky MC, Vaphiades DO. Magnetic resonance imaging in pseudotumor cerebri. Ophthalmology. 1998;105:1686-93.

7. Bateman GA, Stevens SA, Stimpson J. A mathematical model of idiopathic intracranial hypertension incorporating increased arterial inflow and variable venous outflow collapsibility. J Neurosurg. 2009;110:446-56.

8. Butros SR, Goncalves LF, Thompson D, Agarwal A, Lee HK. Imaging features of idiopathic intracranial hypertension, including a new finding: widening of the foramen ovale. Acta Radiologica. 2012;53:682-8.

9. Hoffman J, Huppertz HJ, Schmidt C, et al. Morphometric and volumetric MRI changes in idiopathic intracranial hypertension. Cephalalgia. 2013;33:1075-84. 